\title{
Finite-temperature properties of frustrated classical spins coupled to the lattice
}

\author{
Cédric Weber, ${ }^{1,2}$ Federico Becca, ${ }^{3}$ and Frédéric Mila ${ }^{1}$ \\ ${ }^{1}$ Institute of Theoretical Physics, Ecole Polytechnique Fédérale de Lausanne, BSP, CH-1015 Lausanne, Switzerland \\ ${ }^{2}$ Institut Romand de Recherche numérique sur les Matériaux, Ecole Polytechnique Fédérale de Lausanne, CH-1015 \\ Lausanne, Switzerland \\ ${ }^{3}$ INFM-Democritos, National Simulation Centre, and International School for Advanced Studies (SISSA), I-34014 Trieste, Italy
}

(Received 3 March 2005; revised manuscript received 7 June 2005; published 22 July 2005)

\begin{abstract}
We present extensive Monte Carlo simulations for a classical antiferromagnetic Heisenberg model with both nearest $\left(J_{1}\right)$ and next-nearest $\left(J_{2}\right)$ exchange couplings on the square lattice coupled to the lattice degrees of freedom. The Ising-like phase transition, which appears for $J_{2} / J_{1}>1 / 2$ in the pure spin model, is strengthened by the spin-lattice coupling and is accompanied by a lattice deformation from a tetragonal symmetry to an orthorhombic one. Evidence that the universality class of the transition does not change with the inclusion of the spin-lattice coupling is reported. Implications for $\mathrm{Li}_{2} \mathrm{VOSiO}_{4}$, the prototype for a layered $J_{1}-J_{2}$ model in the collinear regime, are also discussed.
\end{abstract}

DOI: $10.1103 /$ PhysRevB.72.024449

PACS number(s): 75.10.Hk, 75.40.Cx, 75.40.Mg

\section{INTRODUCTION}

The role of frustrating interactions in low-dimensional systems is a very important aspect in the modern theory of magnetism in solids ${ }^{1}$ and molecular clusters. ${ }^{2}$ In particular, the presence of finite-temperature phase transitions in twodimensional systems with continuous spin-rotational symmetry, which are ruled out by a naive interpretation of the Mermin and Wagner theorem, has been clearly documented during the last years and continues to attract much attention because of the variety of phenomena that could be generated at low temperature..$^{3-9}$ The main point is that the presence of frustrating interactions can induce nontrivial degrees of freedom, which may undergo a phase transition when the temperature is lowered. Among the strongly frustrated spin systems, probably one of the most important examples is the antiferromagnetic Heisenberg model on the square lattice with both nearest $\left(J_{1}\right)$ and next-nearest neighbor $\left(J_{2}\right)$ couplings, for which it has recently become certain that a very interesting scenario shows up for large enough frustrating ratio $J_{2} / J_{1}$. Indeed, for $J_{2} / J_{1}>1 / 2$ and classical spins, the two sublattices are completely decoupled at zero temperature, and each of them has an independent antiferromagnetic order. Therefore, the ground-state energy does not depend on the relative orientation between the magnetizations of the two sublattices and the ground state has an $O(3) \times O(3)$ symmetry. At low temperature, thermal fluctuations lift this huge degeneracy by an order by disorder mechanism ${ }^{10}$ and two families of collinear states are entropically selected, with pitch vectors $\mathbf{Q}=(0, \pi)$ and $(\pi, 0)$, respectively. Chandra, Coleman, and Larkin ${ }^{4}$ argued that this fact reduces the symmetry to $O(3) \times Z_{2}$, and that the $Z_{2}$ symmetry is broken at low temperature, giving rise to an Ising-like phase transition at finite temperature. Recently, ${ }^{7}$ it has been possible to verify this scenario by using extensive Monte Carlo simulations, and a rather accurate estimate of the critical temperature as a function of the ratio $J_{2} / J_{1}$ has been obtained.

The fact that the two states with $\mathbf{Q}=(0, \pi)$ and $(\pi, 0)$ break the rotational symmetry, having antiferromagnetic spin correlations in one spatial direction and ferromagnetic correlations in the other, suggests that, once the spin-lattice coupling is considered, the lattice could also experience a phase transition, ferromagnetic and antiferromagnetic bonds acquiring different lengths. Indeed, on general grounds, the super-exchange couplings come from a virtual hopping of the electrons, which depends upon the actual distance of the ions. A first evidence that a similar distortion of the lattice appears when the spins are coupled to classical lattice distortions has been found in Ref. 11, where the quantum $J_{1}-J_{2}$ model at zero temperature has been analyzed by Lanczos diagonalization on small clusters. In particular, by considering a $4 \times 4$ lattice and spin-1/2 coupled to adiabatic phonons, it has been shown that there is a large region where the lattice distorts and ferromagnetic and antiferromagnetic bonds acquire different lengths.

Interestingly, there is also clear evidence that such a scenario is realized in a real compound, $\mathrm{Li}_{2} \mathrm{VOSiO}_{4}$, a vanadate which can be considered as a prototype of the spin-1/2 $J_{1}$ $-J_{2}$ model in the collinear region: ${ }^{12,13}$ Indeed, in this material the Vanadium ions are in the $V^{+4}$ configuration, giving rise to a two-dimensional lattice (weakly coupled in the perpendicular direction) of interacting spin 1/2. Although the value of $J_{2} / J_{1}$ is not exactly known, ${ }^{13-15}$ all estimates indicate that $J_{2} \gtrsim J_{1}$. Moreover, NMR and muon spin rotation magnetization provide a clear evidence for the presence of a phase transition to a collinear order at $T_{c} \sim 2.8 \mathrm{~K}$ and a structural distortion at a nearby temperature. A simple and appealing explanation relies on the existence of the Ising-like transition and the concomitant lattice distortions.

Since an unbiased finite-temperature analysis of the quantum model for large clusters is, at present, impossible, in this work we would like to address the simpler problem of classical spins coupled to lattice distortions, which already represents a highly nontrivial problem. Indeed, for $J_{2} / J_{1}>1 / 2$, where the two families of collinear states are entropically selected, lattice deformations are known to play an important role, ${ }^{11}$ but the effect of the spin-lattice coupling on the nature of the finite-temperature phase transition cannot be predicted by using general arguments and an intensive numerical in- 
vestigation is requested. Indeed, two different scenarios are a priori possible: $(i)$ The Ising-like character of the transition is preserved, and, besides the distortion of the underlying lattice, there is only some quantitative changes in the critical temperature and in some physical quantities like the specific heat. (ii) The spin-lattice coupling radically changes the nature of the original Ising-like transition, giving rise to a firstorder phase transition, with an abrupt change both in spin and lattice properties. Therefore, in this paper we would like to address the problem to understand how the Ising-like transition is modified in the presence of the spin-lattice coupling, by studying both spin and lattice properties.

The paper is organized as follows: In Sec. II, we present the model and the method we used and in Sec. III we show the results and draw the conclusions.

\section{THE MODEL AND THE METHOD}

In this section we introduce the spin-lattice Hamiltonian and briefly describe the method we used to treat the lattice degrees of freedom. The Hamiltonian reads:

$$
\begin{aligned}
\hat{\mathcal{H}}= & \sum_{\langle i, j\rangle} J_{1}\left(d_{i j}\right) \hat{\mathbf{S}}_{i} \cdot \hat{\mathbf{S}}_{j}+\sum_{\langle i, j\rangle\rangle} J_{2}\left(d_{i j}\right) \hat{\mathbf{S}}_{i} \cdot \hat{\mathbf{S}}_{j}+\frac{K_{1}}{2} \sum_{\langle i, j\rangle}\left(\frac{d_{i j}-d_{i j}^{0}}{d_{i j}^{0}}\right)^{2} \\
& +\frac{K_{2}}{2} \sum_{\langle\langle i, j\rangle\rangle}\left(\frac{d_{i j}-d_{i j}^{0}}{d_{i j}^{0}}\right)^{2},
\end{aligned}
$$

where $\hat{\mathbf{S}}_{i}$ are $O(3)$ spins on a periodic square lattice with $N$ $=L \times L$ sites. $\langle i, j\rangle$ and $\langle\langle i, j\rangle\rangle$ indicate the sum over nearest and next-nearest neighbors, respectively. The super-exchange couplings $J_{1}\left(d_{i j}\right)$ and $J_{2}\left(d_{i j}\right)$ depend upon the distance $d_{i j}$ of the sites $i$ and $j$, and $K_{1}$ and $K_{2}$ are the elastic coupling constants. Finally, $d_{i j}^{0}$ is the bare lattice distance. In transition metal compounds, general arguments lead to exchange integrals that vary like the inverse of the distance to a certain power $\theta$, with $\theta$ in the range $5-15 .{ }^{16}$ Therefore, for small displacements around the equilibrium positions, we can write

$$
J\left(d_{i j}\right)=J\left(\frac{d_{i j}^{0}}{d_{i j}}\right)^{\theta} \simeq J\left[1-\theta\left(\frac{d_{i j}-d_{i j}^{0}}{d_{i j}^{0}}\right)\right] .
$$

In the Hamiltonian of Eq. (1), we treat both the spins $\hat{\mathbf{S}}_{i}$ and the lattice coordinates $d_{i j}$ as dynamical variables, allowing them to change their configurations. With respect to the pure spin problem, this fact doubles the number of dynamical variables present in the problem and makes the Monte Carlo algorithm much heavier. In particular, we find it better to use Monte Carlo algorithms with local and/or global updates, instead of more involved Monte Carlo methods based on the reconstruction of the density of states, ${ }^{17,18}$ which we used in the case where the lattice is kept fixed.

In practice, we work on a torus with two independent lengths $L_{x}$ and $L_{y}$, which play the role of additional degrees of freedom sampled by Monte Carlo. Moreover, each site of the cluster can independently change its position in the lattice. These facts allow us to consider structural distortions as well as the usual spin properties. We found that, besides small displacements around their equilibrium positions, the

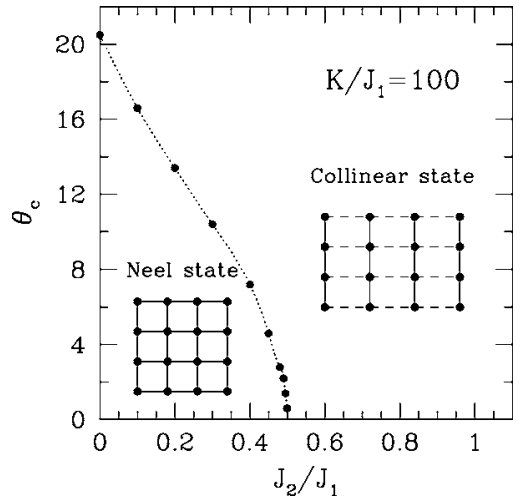

FIG. 1. Zero-temperature phase diagram of the Hamiltonian of Eq. (1): the Néel state, with a tetragonal lattice, is stabilized for $J_{2} / J_{1}<0.5$ and below a critical value of the spin-lattice coupling $\theta_{c}$, otherwise the ground state has collinear spins, with antiferromagnetic correlations along one spatial direction and ferromagnetic along the other, and the lattice has an orthorhombic structure. Lines are guides to the eye.

sites always form either a square cluster, with tetragonal symmetry and equal lattice spacing, $l_{x}$ and $l_{y}$, in the two spatial directions (i.e., $l_{x}=l_{y}$ ), or a rectangular cluster, with orthorhombic symmetry and different lattice spacings in the two directions (i.e., $l_{x} \neq l_{y}$ ). In these cases the total lengths of the cluster in the two directions are $L_{x}=L \times l_{x}$ and $L_{y}=L$ $\times l_{y}$.

For the pure spin model, e.g., with fixed distances and $J\left(d_{i j}\right)=J$, in order to characterize the Ising-like phase transition, it is useful to construct, from the original spin variables $\hat{\mathbf{S}}_{i}$, an effective Ising variable (on the dual lattice) ${ }^{4}$

$$
\sigma_{x}=\frac{\left(\hat{\mathbf{S}}_{i}-\hat{\mathbf{S}}_{k}\right) \cdot\left(\hat{\mathbf{S}}_{j}-\hat{\mathbf{S}}_{l}\right)}{\left|\left(\hat{\mathbf{S}}_{i}-\hat{\mathbf{S}}_{k}\right) \cdot\left(\hat{\mathbf{S}}_{j}-\hat{\mathbf{S}}_{l}\right)\right|},
$$

where $(i, j, k, l)$ are the corners with diagonal $(i, k)$ and $(j, l)$ of the plaquette centered at the site $x$ of the dual lattice. In this way, the two collinear states with $\mathbf{Q}=(\pi, 0)$ and $\mathbf{Q}$ $=(0, \pi)$ can be distinguished by the value of the Ising variable, $\sigma_{x}= \pm 1$. As emphasized in Ref. 7, the normalization term does not affect the critical properties of the model and it is only introduced to have a normalized variable.

Moreover, we can easily construct the Ising-like order parameter as $M_{\sigma}=(1 / N) \Sigma_{x} \sigma_{x}$. Associated to the Ising magnetization $M_{\sigma}$, we can define the susceptibility related to the Ising variable $\chi=(N / T)\left(\left\langle M_{\sigma}^{2}\right\rangle-\left\langle\left|M_{\sigma}\right|\right\rangle^{2}\right)$. Finally, in order to study the finite-temperature phase diagram, it is also useful to consider the specific heat per site $C_{v}=\left(1 / L T^{2}\right)\left(\left\langle E^{2}\right\rangle\right.$ $\left.-\langle E\rangle^{2}\right), E$ being the total energy of the system.

Before considering the Monte Carlo results at finite temperature, it is useful to discuss the zero-temperature phase diagram of the spin-lattice Hamiltonian of Eq. (1) (see Fig. 1). In practice, we have performed Monte Carlo simulations at extremely low temperature, i.e., down to $T / J_{1}=10^{-5}$. In order to be sure to reach the actual minimum of the energy, we have considered different starting configurations for both spins and lattice deformations. In this case, the first-order 
phase transition present for $\theta=0$, i.e., for the pure spin model, at $J_{2} / J_{1}=0.5$ bends towards smaller values of the frustrating ratio $J_{2} / J_{1}$ when the coupling to the lattice is switched on. In particular, the huge $O(3) \times O(3)$ degeneracy is already broken at zero temperature, and the collinear states with pitch vector $\mathbf{Q}=(\pi, 0)$ and $\mathbf{Q}=(0, \pi)$ have a lower energy. It is worth noting that this is a general property of the spin-lattice coupling, which is also present in the quantum case. ${ }^{11}$ Therefore, on the basis of the analysis presented in Ref. 7, in the presence of the spin-lattice coupling, we expect that a finite-temperature phase transition shows up also for a bare ratio $J_{2} / J_{1}<1 / 2$, whenever the coupling $\theta$ is sufficiently large, i.e., $\theta>\theta_{c}$. In the following, we will present strong evidence that there is a finite-temperature phase transition that is related to a change in the lattice symmetry, from a high-temperature disordered magnetic phase with a tetragonal lattice to a low-temperature Ising-ordered phase, with $M_{\sigma} \neq 0$, and with an orthorhombic lattice. A little bit more subtle is the determination of the universality class of this transition. By using powerful Monte Carlo methods, we showed that, in the absence of spin-lattice coupling, the transition belongs to the Ising universality class, ${ }^{7}$ as expected from more general arguments. ${ }^{4}$ The concomitant presence of spin and lattice degrees of freedom makes the Monte Carlo calculation much heavier than in the simpler case where only spins are considered, and we are limited to smaller clusters. Nevertheless, we can reach rather large lattices that enable us to have convincing results for the thermodynamic limit.

\section{RESULTS AND DISCUSSION}

In this section, we present the Monte Carlo results for the Hamiltonian of Eq. (1), and we give evidence that the Ising transition present in the pure spin model survives when the spin-lattice coupling is taken into account, and, moreover, that it is accompanied by a structural deformation.

As discussed in the previous section, in the presence of both spin and lattice degrees of freedom, the Monte Carlo simulations become rather expensive and we are limited concerning the size of the clusters. In order to have a sufficiently high transition temperature that allows a reliable estimate of the physical properties, most of the calculations have been performed for $J_{2} / J_{1}=0.8$ and three different values of the spin-lattice coupling, $\theta=5,10$, and 15 for $K_{1}=K_{2}=K$. In the following we consider the case of $K / J_{1}=100$. Then, we also report calculations for other values of the ratio $J_{2} / J_{1}$, where a similar behavior is observed. Therefore, on the basis of our numerical results, we argue that different choices for the frustrating ratio $J_{2} / J_{1}$ or for the elastic coupling $K_{1}$ and $K_{2}$ do not change qualitatively the physical behavior of the system. However, it should be emphasized that a precise determination of the critical exponents could be difficult and, in general, rather large clusters are needed. In the following, we present evidence that the transition belongs to the Ising universality class, which has been clearly documented in the absence of spin-lattice coupling.

First of all, in order to have some useful insight into the order of the phase transition, we consider Binder's fourth energy cumulant:

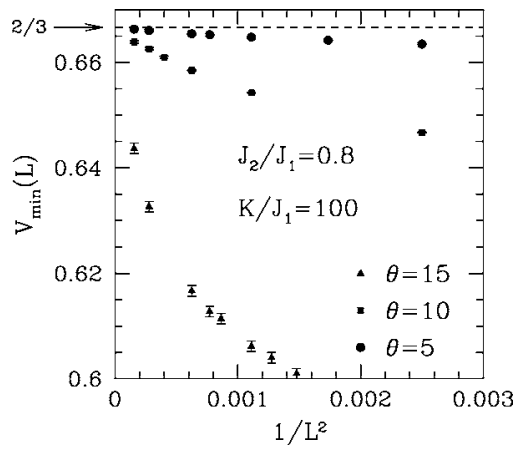

FIG. 2. $V_{\min }(L)$ as a function of $1 / L^{2}$ for $\theta=5,10$, and 15 . The horizontal line, located at $2 / 3$, indicates the value for a continuous phase transition.

$$
V_{L}(T)=1-\frac{\left\langle E^{4}\right\rangle}{3\left\langle E^{2}\right\rangle}
$$

Indeed, by considering the size scaling of $V_{\min }(L)$, the value of $V_{L}(T)$ at the "critical" temperature of the finite cluster $T_{c}(L)$, it is possible to discriminate between a continuous transition, for which $V^{*}=\lim _{L \rightarrow \infty} V_{\min }(L)=2 / 3$, and a firstorder transition, for which $V^{*}<2 / 3 .{ }^{19}$ The size dependence of $V_{L}\left(T_{c}\right)$ is shown in Fig. 2 for three values of $\theta$. Interestingly, in all the cases considered $V_{\text {min }}(L) \rightarrow 2 / 3$, giving a clear evidence that the transition is continuous and not first order.

In Figs. 3 and 4, we show the specific heat and the spin susceptibility $\chi$ for $\theta=5$ and 10 , respectively. In both quantities, the presence of a huge peak, which grows with the size of the cluster, marks the presence of a phase transition. It is worth noting that, while the maximum value of the spin susceptibility is not affected by the spin-lattice coupling, the maximum value of the specific heat strongly depends upon $\theta$. Moreover, the low-temperature value of the specific heat is renormalized by the presence of the lattice displacements: the bare value $C_{v}=1$, which holds when the lattice is frozen in its equilibrium configuration, is changed into $C_{v}=2$ whenever the sites can have small displacements around their equilibrium positions.

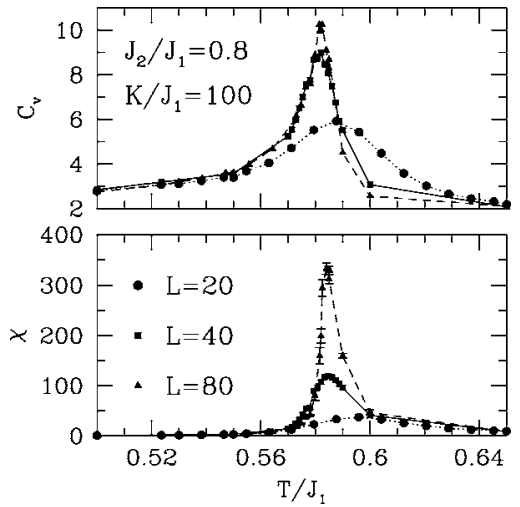

FIG. 3. Upper panel: Specific heat as a function of the temperature for $\theta=5$ for three different clusters $L=20,40$, and 80 . Lower panel: The same for the spin susceptibility. 


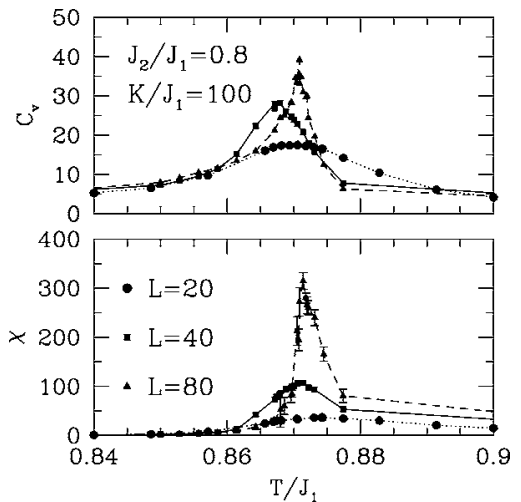

FIG. 4. The same as in Fig. 3 for $\theta=10$.

The actual determination of the universality class of the transition can be assessed by considering the size scaling of the physical quantities. For a second-order phase transition, the maximum value of the susceptibility and of the specific heat follow well-defined scaling relations, i.e., $\chi_{\max }(L)$ $\sim L^{\gamma / \nu}$ and $C_{\text {max }}(L) \sim L^{\alpha}$ (a logarithmic behavior, i.e., $\alpha=0$, for the Ising universality class). On the other hand, for a discontinuous phase transition, we have $\chi_{\max }(L) \sim L^{2}$ and $C_{\text {max }}(L) \sim L^{2}$. As clearly shown for $\theta=0,{ }^{7}$ when the spins are completely decoupled from the underlying lattice, the phase transition is second order and falls into the Ising universality class, i.e., $\alpha=0, \nu=1$, and $\gamma=7 / 4$. In this case, an accurate determination of $\alpha$ is quite hard and rather large clusters are needed (i.e., $L \sim 200$ ). In the case of a finite $\theta$, we cannot afford such large clusters, but, nevertheless, we can obtain rather convincing evidences in favor of the Ising universality class by considering the case of a large spin-lattice coupling $\theta$. Indeed, in Fig. 5, we show the size scaling of the maximum of the specific heat and of the spin susceptibility for $\theta=15$. As mentioned before, the value of the peak of the spin susceptibility does not depend upon $\theta$, and, therefore, the results are also valid for other values of the spin-lattice couplings. A three-parameter fit of $\chi_{\max }(L)=a+b \times L^{\gamma / \nu}$ gives $\gamma / \nu=1.7 \pm 0.1$, which is in reasonable agreement with the value 1.75 of the Ising transition. Moreover, the clearest evi-
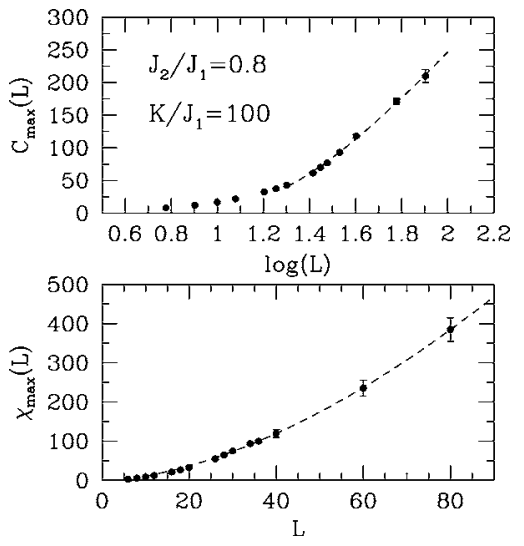

FIG. 5. Upper panel: Size scaling of the maximum of the specific heat as a function of the size of the cluster for $\theta=15$. Lower panel: The same for the maximum of the spin susceptibility. In both cases, the dashed line is a three-parameter fit (see text).

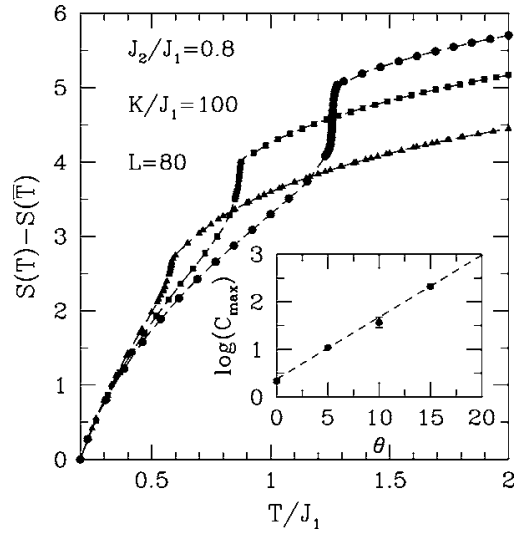

FIG. 6. Entropy difference $S(T)-S(\bar{T})$ as a function of the temperature for $L=80$ and $\theta=5$ (full triangles), 10 (full squares), and 15 (full circles). The reference temperature is $\bar{T} / J_{1}=0.2$. Inset: The maximum of the specific heat for $L=80$ as a function of $\theta$.

dence that the transition belongs to the Ising universality class comes from the specific heat. Indeed, the best fit of the maximum of the specific heat is given by $C_{\text {max }}(L)=a_{0}$ $+a_{1} \log (L)+a_{2} / L,{ }^{20}$ while a power law turns out to be completely inadequate. This fact completely rules out the possibility to have a first-order phase transition, for which a power law with an exponent $\alpha=2$ is expected. For smaller values of $\theta$ it is much harder to get an accurate fit of $C_{\text {max }}(L)$, and much larger sizes should be considered. Nevertheless, having in mind the results for $\theta=0$, we expect that the phase transition also falls into the Ising universality class for small spinlattice coupling. ${ }^{21}$

Although the transition remains of Ising type for all $\theta$, the effect of the spin-lattice coupling on the specific heat is quite large, especially on the actual value of $C_{\max }(L)$. Indeed, by increasing $\theta$, the peak of the specific heat grows exponentially (see inset of Fig. 6) and the transition becomes sharper and sharper, still remaining second order for the values of $\theta$ considered. To get more insight on this point, it is useful to consider the entropy $S(T)$, and in particular its change across the critical temperature. Therefore, starting from the specific heat, we have calculated the entropy per site using the standard formula:

$$
S(T)-S(\bar{T})=\int_{\bar{T}}^{T} d T^{\prime} \frac{C_{v}\left(T^{\prime}\right)}{T^{\prime}},
$$

where $\bar{T}$ is a reference temperature. Notice that, since in a classical system with continuous symmetry the specific heat at zero temperature is finite, it only makes sense to calculate entropy differences between two finite temperatures. Because the width of the peak of the specific heat gets narrow by increasing $\theta$, we expect that the effect of the spin-lattice coupling is much smaller in $S(T)$ than the one in $C_{\max }(L)$. The change of the entropy across $T_{c}$ and its dependence upon the spin-lattice coupling are reported in Fig. 6, where the reference temperature is $\bar{T} / J_{1}=0.2$. In this case, the change of the entropy due to the Ising-like phase transition if proportional to $\theta$, indicating that, although the effect the spin- 


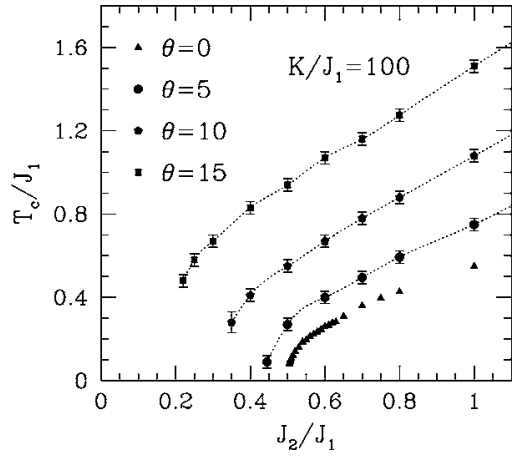

FIG. 7. The critical temperature $T_{c}$ as a function of the frustrating ratio $J_{2} / J_{1}$ for three different values of the spin-lattice couplings, $\theta=5,10$, and 15 . The case for the pure spin model, corresponding to $\theta=0$, is also reported for comparison.

lattice coupling on the peak of $C_{v}$ is huge, its effect on the transition is much less dramatic. This dependence on $\theta$ indicates that, although the critical exponents are unchanged, the transition becomes more and more abrupt when $\theta$ is increased, a feature also observed in the temperature dependence of the magnetization and of the displacements (see below).

By using susceptibility and specific heat measurements for different $J_{2} / J_{1}$, it is possible to determine the behavior of the critical temperature $T_{c}$ as a function of the frustrating ratio $J_{2} / J_{1}$ for different values of $\theta$. The results are reported in Fig. 7. In particular, in order to determine $T_{c}$, we used two alternative methods that give consistent results: On one hand, we take the temperatures for which the susceptibility has its maximum and we make use of the scaling $T_{c}(L) \sim T_{c}+a$ $\times L^{-1}$ (which assumes $\nu=1$ ); on the other hand, we used Binder's fourth cumulant of the Ising parameter. Interestingly, as already anticipated, for a sufficiently large value of the spin-lattice coupling there is a phase transition to a collinear phase even if $J_{2} / J_{1}<1 / 2$ as soon as the spin-lattice coupling is switched on, the critical ratio $J_{2} / J_{1}$ for which a transition occurs for a given $\theta$ being in good agreement with the static analysis (Fig. 1).

Now, we turn to the discussion of the lattice properties. Because the two families of states with pitch vectors $\mathbf{Q}$ $=(0, \pi)$ and $(\pi, 0)$, which are entropically selected, have different spin correlations in the two spatial directions, whenever the sites of the lattices are coupled to the spins, a structural transition is also likely to show up. Indeed, while at high temperature the lattice has a tetragonal symmetry, with the same lattice spacing in the $x$ and $y$ directions, at the transition temperature, the lattice undergoes a structural phase transition towards an orthorhombic symmetry, with different lattice spacings $l_{x}$ and $l_{y}$ (see Figs. 8 and 9). This is exactly equivalent to what happens at zero temperature in the quantum case, where the collinear phase is also accompanied by a lattice distortion. Notice that, since we are considering classical spins, the only relevant distortion is the orthorhombic one, and, indeed, we do not find any evidence towards other displacements of the underlying lattice, in contrast to the spin $1 / 2$ case. $^{11}$

One important outcome is that the structural phase transition has a huge effect just below the critical temperature.

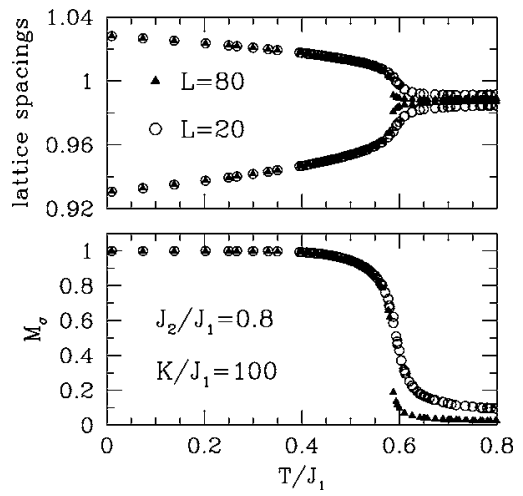

FIG. 8. Upper panel: lattice parameters $l_{x}$ and $l_{y}$ as a function of the temperature for $\theta=5$ for $L=20$ and 80 . Lower panel: The same for the Ising magnetization $M_{\sigma}$.

Indeed, the lattice displacements strongly stabilize one of the two families of states, and almost freeze the spin fluctuations. This can be clearly seen from the Ising-like magnetization $M_{\sigma}$, whose value is practically fixed to its saturation value from zero temperature up to $T_{c}$, where it goes to zero (or to a very small value for finite sizes) (see Figs. 8 and 9).

The implications for quasi-two-dimensional models with interplane magnetic couplings will presumably depend on the value of this coupling. If it is sufficiently weak, we expect the system to undergo two phase transitions: first, coming from high temperature, an Ising-like transition involving a lattice distortion and a breaking of the rotational symmetry of spin fluctuations, then a three-dimensional magnetic ordering. However, since the correlation length increases exponentially fast at low temperature in two-dimensional antiferromagnets, the critical temperature for three-dimensional ordering is expected to grow very fast with the interplane coupling, reaching quite rapidly values of the order of the intraplane couplings. In that situation, the two transitions might very well merge into a single phase transition, with a critical behavior that could be very different from the standard one for magnetic ordering of three-dimensional Heisenberg antiferromagnets though. ${ }^{22}$ Given the lattice sizes available when phonons are included, this is an issue we could not address, however.

From an experimental point of view, the first prototype of the spin-1/2 $J_{1}-J_{2}$ model has been recently synthetized. ${ }^{12}$ It

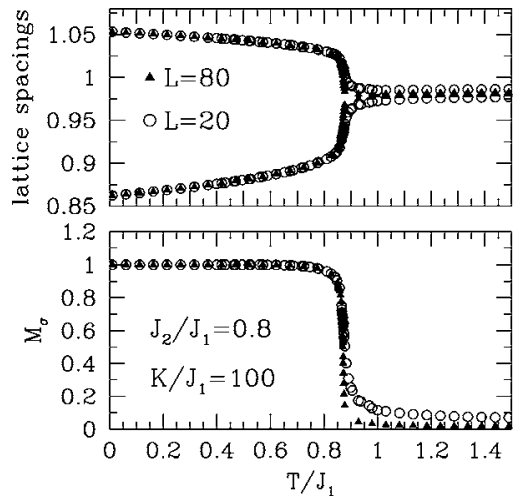

FIG. 9. The same as in Fig. 8 for $\theta=10$. 
is a layered vanadium oxide $\mathrm{Li}_{2} \mathrm{VOSiO}_{4}$ in which $\mathrm{VO}_{5}$ pyramids are arranged in such a way that second vanadium neighbors are in the same plane while first neighbors are not, so that $J_{2}$ need not be smaller than $J_{1}$. Although there is no general agreement on the precise value of the ratio $J_{2} / J_{1}$, both experimental and theoretical estimates lead to a ratio significantly larger than $1 / 2$. Therefore, the system is expected to develop collinear order, which has been confirmed by NMR results at the Li site. Indeed, the NMR spectrum at this site clearly shows that, below $T_{c} \sim 2.8 \mathrm{~K}$, the central peak splits into three different peaks that correspond to different $\mathrm{Li}$ sites where the local field is either zero or nonzero (parallel or antiparallel to the external field). This behavior has been associated to a magnetic order where the spins lie along the $x$ direction with the magnetic vector $\mathbf{Q}=(0, \pi){ }^{13}$ Moreover, the ${ }^{29} \mathrm{Si} \mathrm{NMR}$ spectrum is very anomalous at low temperature $^{13}$ and, given the very symmetric position of Si in the lattice, this cannot be attributed to the development of collinear order but must be related to a structural phase transition. Given the rather low characteristic temperatures in that system $\left(T_{c} \simeq 2.8 \mathrm{~K}\right.$ while the transfer of weight of the $\mathrm{Si}$ line is complete at $2 \mathrm{~K}$ ), a precise structural determination of the low-temperature phase has not been possible yet. Interestingly enough, the temperature at which the system starts to develop a structural distortion seems to be slightly larger than $T_{c}$, which would agree with the general expectation for small interplane coupling. Indeed, experimentally, it is not possible to exclude that distorted regions start to generate above $2.8 \mathrm{~K}$ and grow, by decreasing the temperature, by a nucleation procedure. ${ }^{23}$ Whether a more direct determination of the structural transition will confirm this point remains to be seen.

\section{ACKNOWLEDGMENTS}

We are grateful to P. Carretta and L. Capriotti for useful discussions throughout this project. One of us (F.B.) acknowledges the warm hospitality of EPFL, where this project was initiated. F.B. is supported by COFIN-2004 and by INFM. This work was supported by the Swiss National Fund and by MaNEP.
${ }^{1}$ See for instance, Frustrated Spin Systems, edited by H. T. Diep (World Scientific, Singapore, 2003).

${ }^{2}$ D. Gatteschi, A. Caneschi, L. Pardi, and R. Sassoli, Science 265, 1054 (1994).

${ }^{3}$ C. L. Henley, Phys. Rev. Lett. 62, 2056 (1989).

${ }^{4}$ P. Chandra, P. Coleman, and A. I. Larkin, Phys. Rev. Lett. 64, 88 (1990).

${ }^{5}$ D. Loison and P. Simon, Phys. Rev. B 61, 6114 (2000).

${ }^{6}$ R. R. P. Singh, W. Zheng, J. Oitmaa, O. P. Sushkov, and C. J. Hamer, Phys. Rev. Lett. 91, 017201 (2003).

${ }^{7}$ C. Weber, L. Capriotti, G. Misguich, F. Becca, M. Elhajal, and F. Mila, Phys. Rev. Lett. 91, 177202 (2003).

${ }^{8}$ L. Capriotti, A. Fubini, T. Roscilde, and V. Tognetti, Phys. Rev. Lett. 92, 157202 (2004).

${ }^{9}$ L. Capriotti and S. Sachdev, Phys. Rev. Lett. 93, 257206 (2004).

${ }^{10}$ J. Villain, R. Bidaux, J. P. Carton, and R. Conte, J. Phys. (Paris) 41, 1263 (1980).

${ }^{11}$ F. Becca and F. Mila, Phys. Rev. Lett. 89, 037204 (2002).

${ }^{12}$ P. Millet and C. Satto, Mater. Res. Bull. 33, 1339 (1998).

${ }^{13}$ R. Melzi, P. Carretta, A. Lascialfari, M. Mambrini, M. Troyer, P. Millet, and F. Mila, Phys. Rev. Lett. 85, 1318 (2000); R. Melzi, S. Aldrovandi, F. Tedoldi, P. Carretta, P. Millet, and F. Mila,
Phys. Rev. B 64, 024409 (2001).

${ }^{14}$ H. Rosner, R. R. P. Singh, W. H. Zheng, J. Oitmaa, S. L. Drechsler, and W. E. Pickett, Phys. Rev. Lett. 88, 186405 (2002).

${ }^{15}$ G. Misguich, B. Bernu, and L. Pierre, Phys. Rev. B 68, 113409 (2003).

${ }^{16}$ W. A. Harrison, Electronic Structure and the Properties of Solids (Dover, New York, 1980).

${ }^{17}$ P. M. C. de Olivera and T. J. P. Penna, Braz. J. Phys. 26, 677 (1996); P. M. C. de Olivera, ibid. 30, 195 (2000).

${ }^{18}$ F. Wang and D. P. Landau, Phys. Rev. Lett. 86, 2050 (2001).

${ }^{19}$ M. S. S. Challa, D. P. Landau, and K. Binder, Phys. Rev. B 34, 1841 (1986).

${ }^{20}$ A. Ferdinand and M. E. Fisher, Phys. Rev. 185, 832 (1969).

${ }^{21}$ We cannot exclude that a first- or a second-order phase transition with different exponents appears for some choice of the couplings $\theta, K_{2} / K_{1}$, or $J_{2} / J_{1}$.

${ }^{22}$ P. Peczak, A. M. Ferrenberg, and D. P. Landau, Phys. Rev. B 43, 6087 (1991).

${ }^{23}$ From a combined analysis of the NMR shift $\Delta K$ and of the spin susceptibility $\chi_{s}$, it is possible to extract the hyperfine coupling, which shows a rather clear change between $4.4 \mathrm{~K}$ and $5.2 \mathrm{~K}$ (P. Carretta, private communication). 